\title{
Reinstated JET ICRF ILA: Overview and Results
}

\author{
Pierre Dumortier ${ }^{1,2,}$, ,Frédéric Durodié ${ }^{1}$, Trevor Blackman ${ }^{2}$, Walid Helou $^{3}$, Philippe Jacquet ${ }^{2}$, Ernesto Lerche ${ }^{1,2}$, Igor \\ Monakhov $^{2}$, Craig Noble ${ }^{2}$,Volodymyr Bobkov ${ }^{4}$, Richard Goulding ${ }^{5}$, Michael Kaufman ${ }^{5}$, Dirk Van Eester ${ }^{1}$, and JET \\ Contributors $^{* *}$
}

EUROfusion Consortium, JET, Culham Science Centre, Abingdon, OX14 3DB, UK

${ }^{1}$ LPP-ERM/KMS, TEC partner, Brussels, Belgium

${ }^{2}$ CCFE, Culham Science Centre, Abingdon, United Kingdom

${ }^{3}$ IRFM CEA Cadarache 13108 Saint Paul lez Durance France

${ }^{4}$ Max-Planck-Institut für Plasmaphysik, D-85748 Garching, Germany

${ }^{5}$ Oak Ridge National Laboratory, PO Box 2008, Oak Ridge, United States

\begin{abstract}
The works undertaken to reinstate the JET ICRF ILA are reviewed. The vacuum matching capacitors were replaced, an extensive calibration of all the measurements in the RF circuit was carried out, new simulation tools were created and new control algorithms were implemented for the - toroidal and poloidal - phase control of the array as well as for the matching of the second stage. A review of the contribution of the reinstated ILA to the JET programme during the last campaigns is given showing namely that the new controls allowed extending the range of the operation to lower $(29 \mathrm{MHz})$ and higher $(51 \mathrm{MHz})$ frequencies than previously achieved and allowed more flexible and reliable operation. Operation with coupled power levels up to $2.8 \mathrm{MW}$ and voltages up to $40 \mathrm{kV}$ was achieved. ILA results on plasma are discussed and emphasis is given to the features of interest for ITER.
\end{abstract}

\section{Introduction}

The JET ICRF ITER-Like Antenna (ILA) is a closepacked antenna array composed of four Resonant Double Loops (RDLs) arranged in a 2 toroidal by 2 poloidal array. Each RDL consists of two poloidally adjacent short straps fed through in-vessel matching capacitors from a common vacuum transmission line. Two toroidally adjacent RDLs are fed through a $3 \mathrm{~dB}$ combiner-splitter.

The ILA antenna has been operating essentially at 33 and $42 \mathrm{MHz}$ in 2008-9 until a capacitor failure prevented further operation. The assessment of the results identified that achieving routine and full array operation required a better understanding of the RF circuit, more accurate calibrations of the RF measurements and feedback control algorithms for phase control and second stage matching [1].

\section{Pre-operation work and new features}

Tests on a spare capacitor identified the cause of the capacitor failure in March 2009: a micro-leak caused by the cycle wear of the capacitor's internal bellows appears after about 4500 cycles degrading the capacitor's private vacuum. All capacitors were subsequently replaced and a new operating scheme minimizing the number of fullstroke requests was adopted. All capacitors were tested prior to installation and reconditioned if necessary [2].

The $3 \mathrm{~dB}$ combiner-splitters feeding two toroidally adjacent RDLs were removed in 2008-9 due of phase control stability issues [1]. The new algorithms described in Section 3 allow operating with the $3 \mathrm{~dB}$ combinersplitters in the circuit without any phase control stability issue. They moreover provide an isolation of the generators from the rest of the circuit resulting in a much more stable operation of the generators.

The Transmission Line (TL) layout was modified in order to allow operation of the antenna in toroidal dipole $(0 \pi)$ as in Current Drive (CD $-0-\pi / 2)[3]$.

A thorough calibration campaign of all RF quantities has been undertaken: all directional couplers in the TL system, all voltage probes in the antenna [4], all the RF cables and all the electronics modules and RF components have been calibrated and the necessary adaptors characterised $[2,3]$. This allowed achieving the level of accuracy required for the control of the antenna (see Section 3) and the operation of the S-Matrix Arc Detection (SMAD) protection system.

\footnotetext{
* Corresponding author: pierre.dumortier@rma.ac.be

${ }^{* *}$ See the author list of "Overview of the JET results in support to ITER" by X. Litaudon et al. to be published in Nuclear Fusion Special issue: overview and summary reports from the 26th Fusion Energy Conference (Kyoto, Japan, 17-22 October 2016)
} 
Several other improvements were implemented including a full integration of the control system in Level 1 (RF pilot interface), an improved capacitor thermal protection in the RF Local Manager, a revised protection limiting the voltage on the capacitors and the recommissioning and improvement of the SMAD [2].

A low-power commissioning of the ILA using a network analyser was performed before the high power commissioning in order to determine the initial matching settings for the different operating frequencies and chosen conjugate-T impedances $\left(Z_{T}\right)$. This also allowed testing and optimising the control algorithms.

Finally, new numerical modelling and simulation tools were developed, which were used in the calibration of the voltage probes as well as in the implementation of the new control algorithms [4-6].

\section{New control algorithms [6]}

Fig. 1 displays a schematic of the ILA circuit illustrating the feedback control loops.

The first stage matching controls the 4 complex conjugate-T point impedances $\left(Z_{T}\right)$ by adjusting the 8 invessel variable capacitors in each RDL branch (4 feedback loops; 8 controlled parameters). $Z_{T}$ is estimated from the Antenna Pressurised Transmission Line (APTL) forward and reflected directional couplers measurements using a CST MicroWave Studio ${ }^{\circledR}$ [7] model of the APTL with the vacuum ceramic window, the bellows and the transition between the $30 \Omega$ circular feed and the quarter-wave transformer rectangular section whereas it was previously estimated using a TL model of this section. The revised control algorithm was implemented using Field-Programmable Gate Array (FPGA) technology.

No Second-Stage Matching (SSM) control algorithm was implemented in 2008-9 leading to a very tedious procedure of manual adjustments between pulses and no real-time control to adjust to the varying plasma conditions. A new algorithm was implemented, which controls the lengths of the 4 SSM stubs and of the 4 SSM trombones (4 feedback loops; 8 controlled parameters). To optimize the matching conditions in ELMy plasmas, the algorithm has been designed to work on 2 time scales: a fast one $(2 \mu \mathrm{s})$ and a slow one ( 2000 points on the pulse length, i.e. $5 \mathrm{~ms}$ for a $10 \mathrm{~s}$ pulse). On the fast time scale, fast enough to track the coupling variations during the ELMs, the Main Transmission Line (MTL) complex reflection coefficients are processed and recorded onto a polar map divided in 8 phase bins and 15-20 amplitude bins; the points are kept in the bins for a certain time before being removed. On the slow time scale, the average reflection coefficient is estimated from the polar reflection map; the normalised admittance at the MTL side of the SSM stub is then calculated, from which error signals used to drive the SSM stub and trombone are generated. This results in an effective offset match that minimizes the positive Voltage Standing Wave Ratio (VSWR) excursion during an ELM cycle (rather than the inter-ELM VSWR value). The algorithm outputs three parameters: move if the element

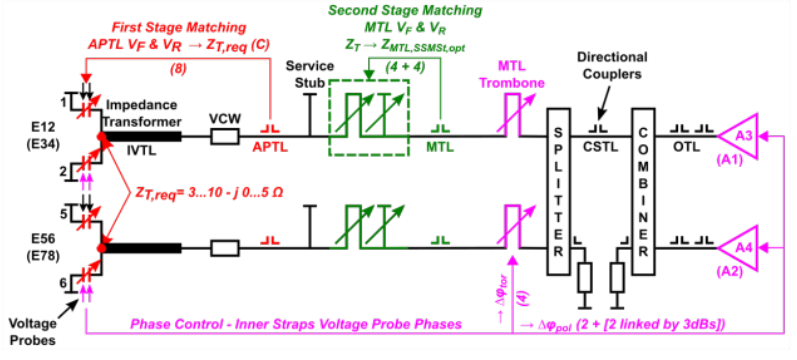

Fig. 1. Schematic of the ILA circuit for the ILA upper half (2 toroidal RDLs) illustrating the feedback control loops. The bottom half circuit is similar but not represented for clarity.
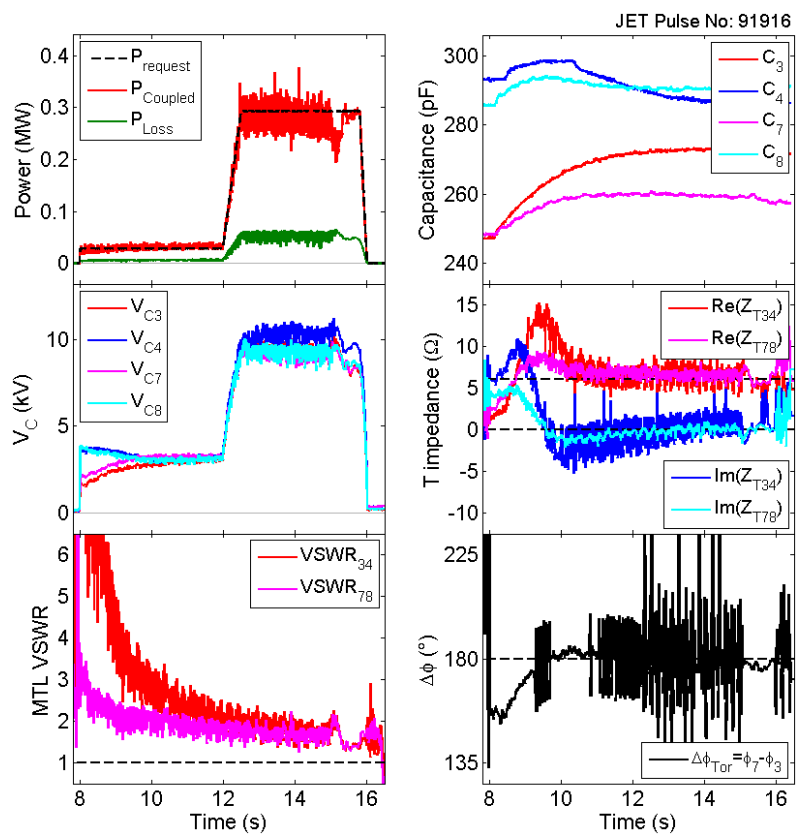

Fig. 2. Example of feedback control on a JET pulse (bottom half array operation). Top left: power request, coupled power and power losses. Middle left: capacitor voltages. Top right: capacitor values actuated by the first stage matching to achieve the requested $Z_{T}=6+0 \mathrm{j} \Omega$ (middle right graph). Bottom left: the SSM actuates the SSM stub and trombones to bring the MTL VSWR down towards an offset matched condition. Bottom right: the toroidal phase feedback actuates the MTL trombones to obtain the requested toroidal phasing (dipole phasing $-180^{\circ}$ in this case).

(stub or trombone) error signal is above a threshold, direction of movement depending on the sign of the error signal and fast that will be true if the amplitude of the signal is above a certain value and will cause a fast movement of the element. The algorithm was extensively simulated [5] and proved stable with respect to the other control algorithms (first stage matching and phase control).

The phasing of the antenna array is controlled by the phase of the voltage probe signals of the four inner straps.

The phase difference between toroidally adjacent RDLs is controlled by acting on the 4 MTL trombones located at the output of the $3 \mathrm{~dB}$ combiner-splitters (2 feedback loops; 4 controlled parameters).

The phase difference between poloidally adjacent RDLs is controlled with respect to a separate phase reference and is adjusted by acting on the 4 generators' 
output phases. Two generators are feeding one $3 \mathrm{~dB}$ combiner-splitter thereby constraining the two generators' output phases (poloidal phasing: 2 feedback loops; 2 independent parameters).

The matching and phasing of the whole array is consequently controlled by 12 feedback loops actuating 22 parameters: the 8 variable in-vessel capacitors, the 4 SSM trombones, the 4 SSM stubs, the 4 MTL trombones and the 4 generator output phases constrained by the 2 $3 \mathrm{~dB}$ combiner-splitters.

Fig. 2 illustrates the operation of the feedback controls during a pulse (bottom half array operation). The pulse starts with a low power phase to home the capacitors in and achieve a reasonable match while staying below the VSWR arc detection trip level. The power is then raised towards the requested value.

The efficiency of the SSM algorithm to optimise the match for the ELM-resilience has been discussed in [6].

\section{Overview of operation results}

The new control algorithms, in particular the SSM control algorithm, together with the improved modelling and a comprehensive calibration of all RF signals have significantly eased the operation of the ILA and allowed to extend the frequency range of the operation towards lower $(29 \mathrm{MHz})$ and higher $(49 \mathrm{MHz}$ for the full array, $51 \mathrm{MHz}$ for the upper half) frequencies. Commissioned frequencies are: $29,33,37,42,44,47,49$ and $51 \mathrm{MHz}$. It also allowed for the first time operation in toroidal CD $(0$ $-\pi / 2$ ) phasing besides the usual toroidal dipole phasing operation $(0 \pi)$. CD operation however gives rise to voltage imbalance between the currents and voltages of the two toroidal RDLs due to enhanced mutual coupling [2], which eventually also impacts on the radiated power spectrum. Fig. 3 illustrates a toroidal phase scan from $\mathrm{CD}$ to dipole during a pulse showing the voltage imbalance during $\mathrm{CD}$ operation and voltage balance when reaching dipole operation (low power phase for matching from 8 to $10 \mathrm{~s}$ ). The mutual coupling also generates an imbalance in the reflection coefficients in two toroidal MTLs leading to a higher power diverted to the load of the corresponding $3 \mathrm{~dB}$ hybrid splitter. The levels however remain small (1-3\% for CD phasing).

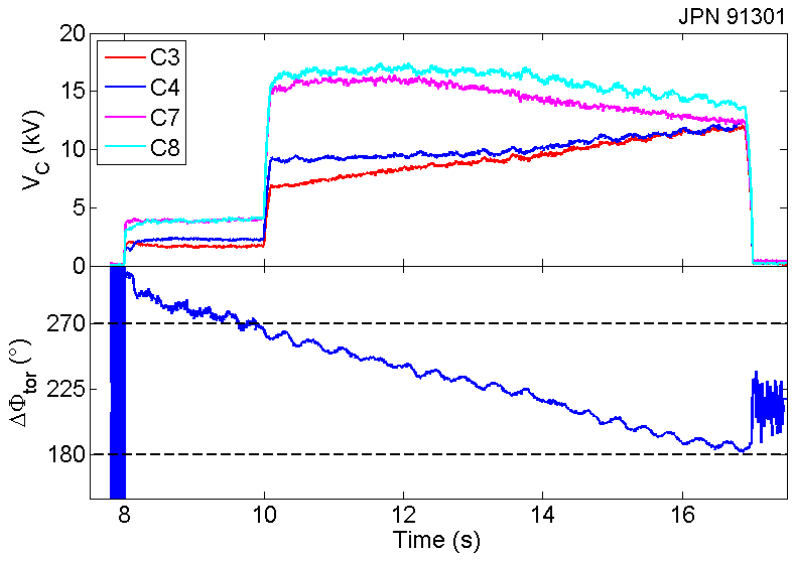

Fig. 3. Toroidal phase scan (top) and its impact on the strap voltage balance (bottom).
The ILA has significantly contributed to the experimental programme of JET C35-C37 campaigns up to power levels of $2.8 \mathrm{MW}$ coupled power (L-mode half array) and about $40 \mathrm{kV}$ maximum voltage. It has been particularly useful at the lower frequencies where the power delivered by conventional A2 antennas is limited by the poor coupling to the plasma and operation limited by the maximum allowed voltage [8]. At $33 \mathrm{MHz}$, half ILA array is able to deliver as much power as 2 A2s, which represents a ten times higher power density.

The operation of the ILA in 2008-9 in JET with a carbon wall has demonstrated that the heating efficiency and the impurity production of the ILA were similar to the ones of the A2s despite the higher power density $[1,9]$. First indications from the operation of the ILA in JET with the ITER-like wall show likewise that there are no adverse effects arising from the higher power density. Fig. 4 shows an example of such a comparison, where $2 \mathrm{MW}$ of ICRH power was coupled from the ILA (full array), antennas $\mathrm{A}+\mathrm{B}(3 \mathrm{~dB}$ system) and antennas $\mathrm{C}+\mathrm{D}$ (external conjugate-T) consecutively in an L-mode plasma with $B_{t}=2.7 \mathrm{~T}, I_{p}=1.8 \mathrm{MA}, \mathrm{H}$ minority heating at $42 \mathrm{MHz}$ (dipole phasing), fixed antenna-plasma distance and constant top gas injection throughout the discharge. This is further supported by investigation of the RFenhanced plasma-surface interactions due to nearantenna electric fields ([10-11]) and impurity (W and Ni) concentration measurements [12]. The impact of the impurity production on the operation of next-step devices such as ITER is further discussed in [12].

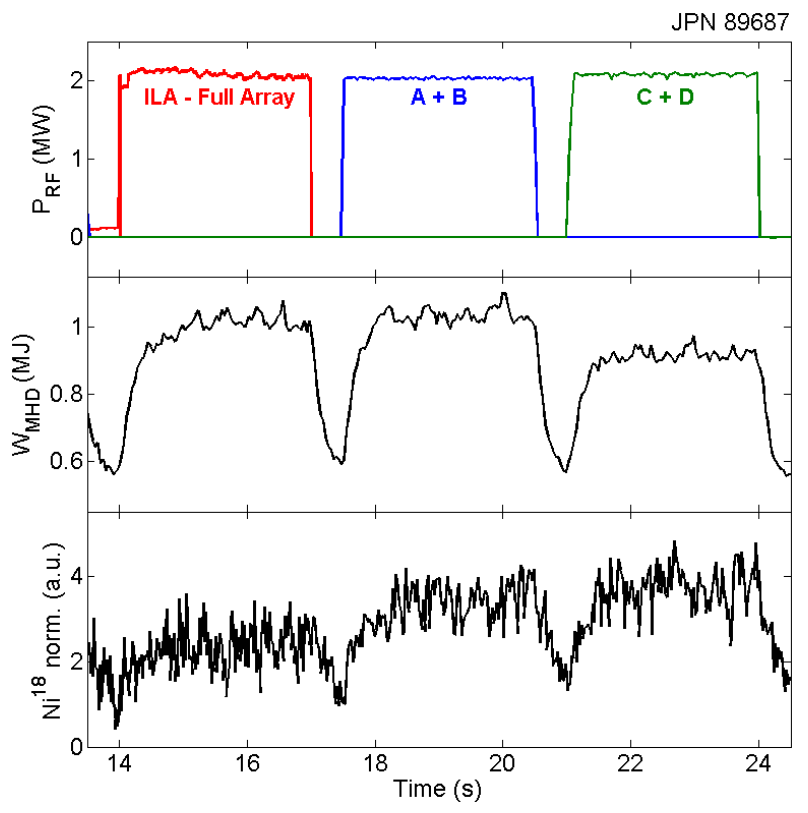

Fig. 4. Comparison between the ILA and the conventional A2 antennas: A+B (fed by $3 \mathrm{~dB}$ hybrid splitters) and $\mathrm{C}+\mathrm{D}$ (fed through an external conjugate-T). Top: coupled power, middle: plasma stored energy, bottom: $\mathrm{Ni}^{18}$ line emission normalised to the plasma edge density.

The coupling resistance for the ILA is defined per RDL as follows:

$$
R_{C x y}=2 P_{\text {coupled } x y} /\left(I_{x}^{2}+I_{y}^{2}\right)
$$


where $x$ and $y$ are the strap numbers $(1 \ldots 8), x y$ is the corresponding RDL number, $P_{\text {Coupled } x y}$ is the power coupled to the plasma by the RDL $x y$ (calculated from the MTL power by subtracting the estimated losses) and $I_{x}, \quad I_{y}$ are the strap currents estimated from the corresponding capacitor voltage probe. The voltage probes are located at the level of the variable capacitors [4] and an equivalent transmission line circuit determined by numerical modelling is used to relate the measured voltage to the strap short circuit current.

Fig. 5 shows the coupling resistance $\left(R_{C}\right)$ variation with the average distance between the strap and the last closed magnetic surface for L-mode discharges with $I_{p}=1.8-2 \mathrm{MA}, B_{t}=2.7 \mathrm{~T}$ and $f=42 \mathrm{MHz}$. The dashed curves correspond to the fit of the coupling resistance of RDL12 for comparison. The scatter in $R_{C}$ is due to the different operating conditions (gas fuelling rate and location, etc.), which can have a significant impact on $R_{C}$. As the heat loads were below the acceptable limits the antenna was moved towards the plasma by $7 \mathrm{~mm}$ (from pulse 89904 on) in order to improve the coupling. The closed/open symbols correspond to data from pulses respectively before/after the $7 \mathrm{~mm}$ shift. The diamonds correspond to operation with the outer strike point of the plasma located on the bulk $\mathrm{W}$ divertor and featuring low pumping (Tile 5). The squares correspond to operation with the outer strike point on the $\mathrm{W}$-coated divertor close to the pumping throat (Tile 6). Fig. 5 shows that all the RDLs have comparable coupling resistances for operation on Tile 6 and that the bottom-half is more sensitive to changes in the Scrape-Off Layer (SOL) conditions, as seen by the somewhat larger spread of the data. With Tile 5 operation the top-half coupling resistances are only slightly modified but the coupling of the bottom RDLs is substantially better than with Tile 6 . This is attributed to the fact that the pumping efficiency is lower when the strike point is on Tile 5 and the density profiles in the far-SOL can be considerably enhanced, in particular below mid-plane.

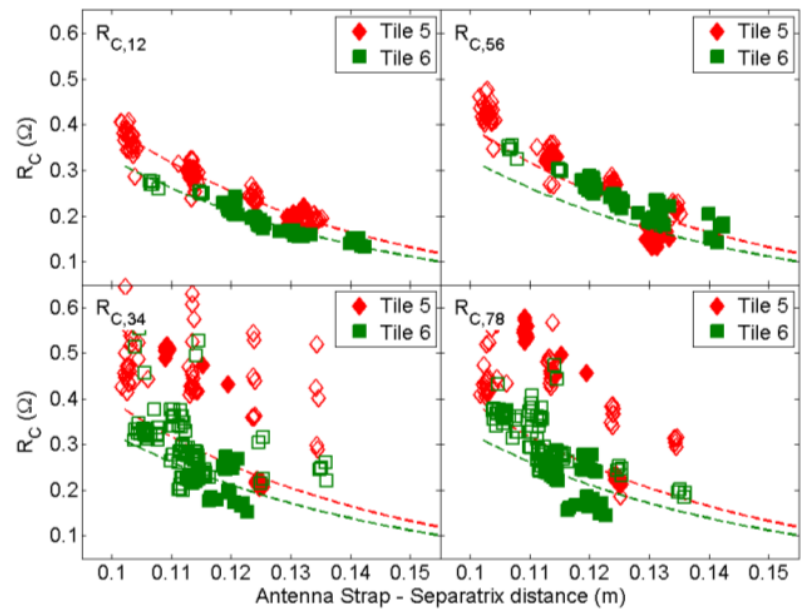

Fig. 5. Coupling resistance vs antenna strap-separatrix distance for the different RDLs.

Whilst the poloidal phase control was not yet fully commissioned most of the pulses during the JET campaigns were operated with half (upper or lower) array. A hydraulic fluid leak at one of the capacitor actuators unfortunately occurred in July 2016. The issue was first identified as a low voltage arc [2] but further investigation identified that the hydraulic fluid eventually flooded the pressure box of RDL56 and part of the corresponding APTL section causing arcing at a ceramic support in the APTL. It also damaged one of the position sensors. This prevented further operation of the upper half of the ILA and hence progress on the commissioning of the full array phase control. Remedial actions are currently being undertaken, which, if successful, would bring the upper half back into operation. Limited but promising results were however achieved for the full ILA array phase control.

\section{Conclusions}

The reinstated ILA has significantly contributed to the experimental programme of the JET C35-C37 campaigns (typically $20-40 \%$ of the total RF power). Improved numerical modelling, tighter calibration of all RF signals and new algorithms have greatly eased the operation, increased the flexibility and improved the reliability of the ILA. The frequency range has been extended towards lower $(29 \mathrm{MHz})$ and higher $(51 \mathrm{MHz})$ frequencies. Dipole and current drive toroidal phasing have been operated.

Feedback control of the two matching stages and of the toroidal phase was routinely used during operation on half array (5 feedback loops; 10 actuators). Limited but promising results were obtained for the control of the full array (12 feedback loops; 22 actuators).

The main features of interest of the ILA for ITER, which have already been demonstrated in JET with the carbon wall [1], have been confirmed in JET featuring the ITER-Like Wall, in particular the operation of a high power density close-packed array antenna without degradation of the performances (coupling, heating efficiency, impurity production) with voltages up to $42 \mathrm{kV}$ (corresponding to $2.5 \mathrm{kV} / \mathrm{mm}$ electric fields).

The issues encountered by the ILA are related to the internal matching components (capacitor bellow in 2009 and hydraulic fluid leak in the capacitor actuator in 2016), which validates the choice of external matching elements for ITER.

This work has been carried out within the framework of the EUROfusion Consortium and has received funding from the Euratom research and training programme 2014-2018 under grant agreement No 633053. The views and opinions expressed herein do not necessarily reflect those of the European Commission.

\section{References}

1. F. Durodié et al., Plasma Phys. Contr. Fus. 54, 074012 (2012).

2. P. Dumortier et al., 'Commissioning and first results of the reinstated JET ICRF ILA', accepted for publication in Plasma Phys. Contr. Fus.; http://dx.doi.org/10.1016/j.fusengdes.2017.05.050 
3. P. Dumortier et al., AIP Conf. Proc. 1689, 070003 (2015); http://doi.org/10.1063/1.4936510.

4. W. Helou et al., Rev. Sci. Instr. 87, 104705 (2016).

5. F. Durodié et al., AIP Conf. Proc. 1689, 070013 (2015); http://doi.org/10.1063/1.4936520

6. F. Durodié et al., 'ITER-Like antenna for JET first results of the advanced matching control algorithms', accepted for publication in Plasma Phys. Contr. Fus. http://dx.doi.org/10.1016/j.fusengdes.2017.05.113.

7. CST GmbH, CST Microwave Studio, User Manual.

8. I. Monakhov et al., this conference.

9. A Czarnecka et al., Plasma Phys. Contr. Fus. 54, 074013 (2012).

10. C.C. Klepper et al., this conference.

11. A. Krrivská et al., this conference.

12. P. Jacquet et al., this conference. 